\title{
Assessment of MRI parameters for studying brain development in newborns with congenital heart disease
}

\author{
Brahmdeep S Saini ${ }^{2 *}$, Prakash Muthusami ${ }^{1}$, Sujana Madathil ${ }^{2}$, Jessie Mei Lim², Christopher Macgowan³, \\ Steven Miller ${ }^{4}$, Mike Seed ${ }^{2}$ \\ From 18th Annual SCMR Scientific Sessions \\ Nice, France. 4-7 February 2015
}

\section{Background}

Microstructural evidence of white matter (WM) dysmaturation in the brains of newborns with congenital heart disease (CHD) using MRI has been previously shown [1]. Further, delayed WM development confers an increased risk of WM injury before and after neonatal cardiac surgery [2]. WM injury is associated with a high incidence of subsequent neurodevelopmental deficits and fetal interventions to improve brain development in the setting of $\mathrm{CHD}$ are currently being investigated. We wished to compare the utility of our previously described parameters in another cohort of CHD newborns, and assess the performance of a new parameter (WM T2 relaxation) for discerning differences in WM maturation.

\section{Methods}

We studied the newborn brains of 30 normal and 21 CHD subjects between June 2013 and September 2014 as part of a hospital IRB approved study. MRI was performed without sedation at a mean age of 7 days (range 0-42 days) on a Siemens Avanto 1.5T system (Erlangen) with the following sequences: high resolution 3D T2W FSE, multivoxel proton magnetic resonance spectroscopy and diffusion tensor imaging. T2 mapping was performed in 6 normal and 6 CHD newborns. We calculated brain volume by segmenting the 3D T2W images using Mimics (Materialise, Leuven). The $\mathrm{N}$-acetyl acetate to choline (NAA/Chol) ratio was calculated from the MRS of the centrum semiovale. Regions of interest for analysis of T2, fractional anisotropy (FA) and apparent diffusion coefficient (ADC) included inferior frontal, superior frontal and parietal
WM. An unpaired t-test was used to determine the statistical significance of differences between the two groups.

\section{Results}

There was no significant difference between the corrected gestational ages of the two groups $(\mathrm{p}=0.88)$. Brain volume increased with age but was lower in CHD newborns than controls (Fig. 1A). The ADC values decreased with age but were higher in CHD newborns than in controls (Fig. 1B). FA and NAA/Chol ratios both increased with age but were not significantly different between the two groups. T2s decreased with age and the average WM T2s of CHD newborns were higher than controls. The T2 brain maps of CHD newborns showed visual differences in comparison to controls of similar age (Fig. 1C-D). Table 1 summarizes all the results.

\section{Conclusions}

As expected, WM ADC values in CHD newborns were significantly higher than controls. We also found a reduction in brain volume in newborns with $\mathrm{CHD}$, similar to the results of other groups [3]. In unmyelinated WM regions, FA and NAA/Chol ratios were not significantly different. Whereas, WM T2 was significantly higher in CHD newborns, despite the smaller number of studies that incorporated T2 mapping. WM T2 may be a sensitive marker of WM dysmaturation in the setting of CHD and a useful adjunct to more established parameters in the assessment of the impact of fetal interventions on brain development. 


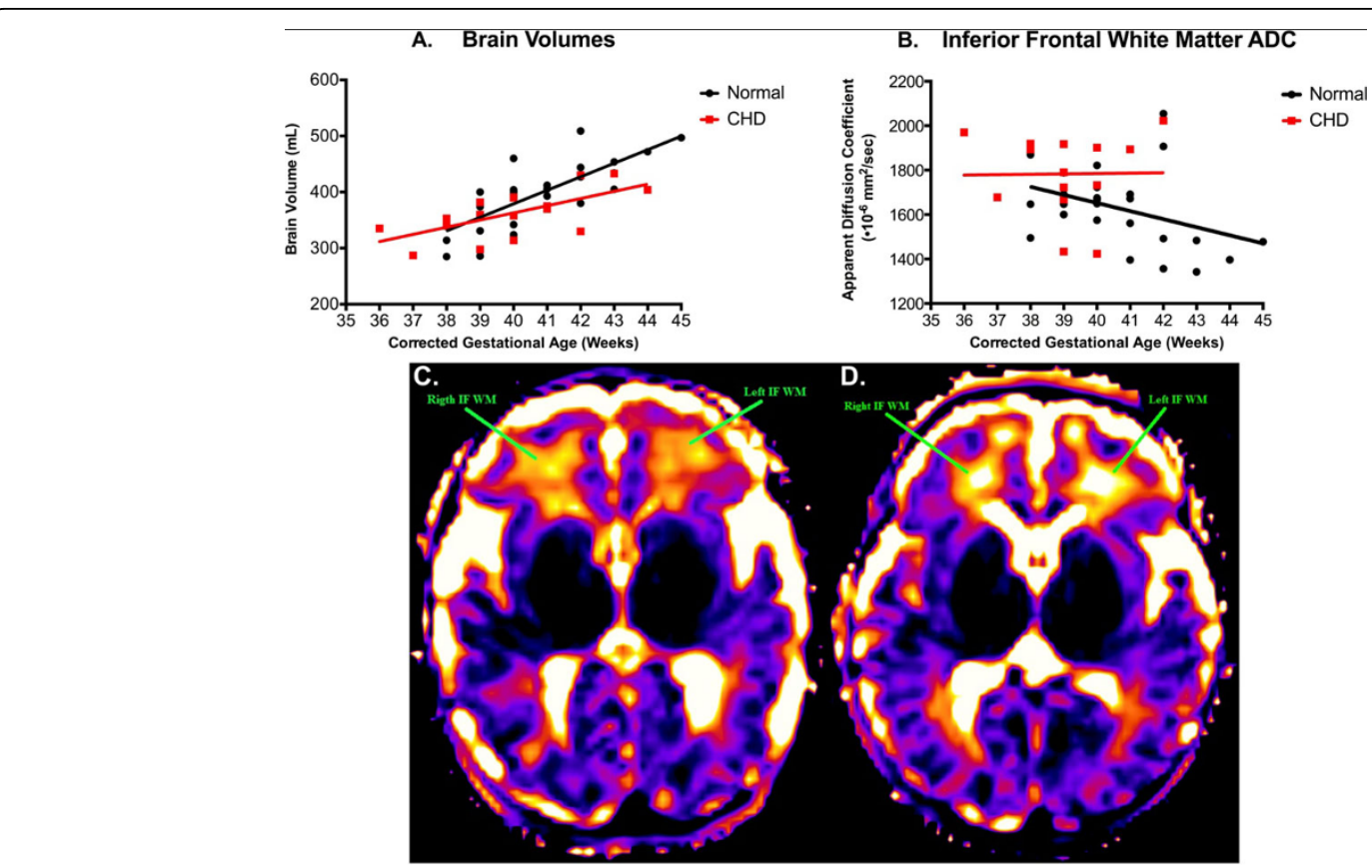

Figure 1 Comparison of brain volumes (A.) and inferior frontal WM ADC (B.) versus corrected gestational age (GA) between CHD newborns and controls. Visual comparison of brain T2 maps at the same window level of inferior frontal WM in a control (C.) to a CHD newborn (D.). Both the normal and CHD newborn were of the same age, 39 weeks corrected GA.

Table 1 Comparison of MRI parameters in assessing brain development between normal and CHD newborns

\begin{tabular}{|c|c|c|c|c|}
\hline MRI Parameter & Control Mean \pm SD & CHD Mean \pm SD & P Value & Significant $(\mathrm{Y} / \mathrm{N})$ \\
\hline Brain Volume $(\mathrm{mL})$ & $392 \pm 57, n=29$ & $362 \pm 41, n=18$ & 0.04 & Y \\
\hline \multicolumn{5}{|l|}{$\begin{array}{c}\text { MRS: } \\
\text { Centrum Semiovale NAA/Chol Ratio }\end{array}$} \\
\hline WM T2 (ms) & $269 \pm 27, n=6$ & $293 \pm 39, n=6$ & 0.04 & Y \\
\hline $\begin{array}{c}\text { Fractional Anisotropy }\left(\times 10^{-3}\right): \\
\text { Inferior Frontal WM } \\
\text { Superior Frontal WM } \\
\text { Parietal WM }\end{array}$ & & & & \\
\hline $\begin{array}{c}\text { Apparent Diffusion Coefficient }\left(\times 10^{-6} \mathrm{~mm}^{2} / \mathrm{sec}\right) \text { : } \\
\text { Inferior Frontal WM } \\
\text { Superior Frontal WM } \\
\text { Parietal WM }\end{array}$ & & & & \\
\hline
\end{tabular}

MRS - magnetic resonance spectroscopy, WM - white matter.

Authors' details

'Diagnostic Imaging, The Hospital for Sick Children, Toronto, ON, Canada. ${ }^{2}$ Heart Centre, The Hospital for Sick Children, Toronto, ON, Canada.

${ }^{3}$ Physiology and Experimental Medicine, The Hospital for Sick Children, Toronto, ON, Canada. ${ }^{4}$ Neurology, The Hospital for Sick Children, Toronto, ON, Canada.

Published: 3 February 2015

\section{References}

1. Miller, et al: N Eng J Med 2007.

2. Dimitropoulos, et al: Neurology 2013.

3. Ortinau, et al: J Thorac Cardiovasc Surg 2012.
doi:10.1186/1532-429X-17-S1-P205

Cite this article as: Saini et al:: Assessment of MRI parameters for studying brain development in newborns with congenital heart disease. Journal of Cardiovascular Magnetic Resonance 2015 17(Suppl 1):P205. 